\title{
Discrete Autocorrelation-Based Multiplicative MRAs and Sampling on $\mathbb{R}$
}

\author{
John J. Benedetto \\ Norbert Wiener Center \\ Department of Mathematics, University of Maryland \\ College Park, MD 20742, USA \\ jjb@math.umd.edu \\ Somantika Datta \\ Department of Mathematics, University of Idaho \\ Moscow, ID 83844-1103, USA \\ sdatta@uidaho.edu
}

\begin{abstract}
Given $K>0$ and a positive integer $M$, the function $\triangle_{K, M}(t)=K \max (1-$ $\left.\frac{|t|}{M}, 0\right)$ represents an isosceles triangle on the real line $\mathbb{R}$ having height $K$ and base $2 M$. For a given $K$ and $M$, a constant amplitude sequence is constructed on the integers $\mathbb{Z}$ having autocorrelation $\triangle_{K, M}$ restricted to $\mathbb{Z}$. With this setup, a large class of bounded sequences $z$ is shown to exist, where the autocorrelation of $z$ is a finite sum of such $\triangle_{K, M} \mathrm{~s}$. By taking an inverse Fourier transform we are led naturally to expansions in terms of Fejér functions. This line of thought is closely related to the construction of constant amplitude zero autocorrelation (CAZAC) sequences which have applications in radar and communications. Extending the setting to $\mathbb{R}$, a Fejér function decomposition formula is obtained for a class of even continuous functions. This gives rise to a decomposition theorem along the lines of what can be described as multiplicative multiresolution analysis (MRA) and sampling theory. The underlying techniques generalize to other generating functions besides the Fejér function, even for the case of $\mathbb{R}$.
\end{abstract}

Key words and phrases: Autocorrelation, Fejér function, multiresolution analysis

2000 AMS Mathematics Subject Classification - 42A38, 42C40, 94A12, $94 \mathrm{~A} 20$ 


\section{Introduction}

\subsection{Goal}

We shall introduce a notion which could aptly be called multiplicative multiresolution analysis (MRA), where the translation and dilation of an MRA are replaced by multiplication and dilation. There is a natural sampling theory associated with this notion. The motivation for this approach is the importance of constructing constant amplitude zero autocorrelation (CAZAC) sequences in a variety of applications, see Section 1.3 and Section 2. The setting herein is classical Fourier analysis on $\mathbb{R}$; but we realistically envision transporting and generalizing the methods of this paper to a more general setting, see Section 1.4 for a hint of how this will be effected.

\subsection{Notation}

We shall use the standard notation from harmonic analysis, e.g., [3], [25]. $\mathbb{N}$ is the set of natural numbers and $\mathbb{C}$ is the set of complex numbers. $C\left(\mathbb{T}^{d}\right)$ is the space of $\mathbb{C}$-valued continuous functions on $\mathbb{T}^{d}=\mathbb{R}^{d} / \mathbb{Z}^{d}$, and $A\left(\mathbb{T}^{d}\right)$ is the subspace of absolutely convergent Fourier series. $M\left(\mathbb{T}^{d}\right)$ is the space of bounded Radon measures on $\mathbb{T}^{d}$, i.e., $M\left(\mathbb{T}^{d}\right)$ is the dual space of the Banach space $C\left(\mathbb{T}^{d}\right)$ taken with the sup norm. For a given $\lambda>0$, the $L^{1}$-dilation of $f, f_{\lambda}$, is defined as $f_{\lambda}(t)=\lambda f(\lambda t)$. Let $\triangle(t)=\max (1-|t|, 0)$ on $\mathbb{R}$. Let $\omega(\gamma)=\frac{1}{2 \pi}\left(\frac{\sin \gamma / 2}{\gamma / 2}\right)^{2}$; $\omega$ is the Fejér function. The Fourier transform of $f \in L^{1}(\mathbb{R})$ is the function $\widehat{f}$ defined by

$$
\widehat{f}(\gamma)=\int_{-\infty}^{\infty} f(t) e^{-2 \pi i t \gamma} d t, \gamma \in \widehat{\mathbb{R}}(=\mathbb{R})
$$

$A(\widehat{\mathbb{R}})$ denotes the space of such absolutely convergent Fourier transforms on $\widehat{\mathbb{R}}$, with an analogous definition for $A\left(\widehat{\mathbb{R}}^{d}\right)$. We write the pairing between the function $f$ and $\widehat{f}$ as $f \leftrightarrow \widehat{f}$. The Fourier transform of $\triangle$ is $\omega_{2 \pi}$. For a set $E$, the measure of $E$ is denoted by $|E|$.

Definition 1.1. The autocorrelation $A_{x}: \mathbb{Z} \rightarrow \mathbb{C}$ of $x: \mathbb{Z} \rightarrow \mathbb{C}$ is formally defined as

$$
\forall k \in \mathbb{Z}, \quad A_{x}[k]=\lim _{N \rightarrow \infty} \frac{1}{2 N+1} \sum_{m=-N}^{N} x[k+m] \overline{x[m]} .
$$

(Lower case Roman letters, such as $x$, are often used in some applied communities to denote functions $\mathbb{Z} \rightarrow \mathbb{C}$.) There is an analogous definition of autocorrelation for functions $f: \mathbb{R}^{d} \rightarrow \mathbb{C}$, e.g., see Theorem 1.2.

If $F \in A\left(\mathbb{T}^{d}\right)$ we write $\check{F}=f=\left\{f_{k}\right\}$, i.e., $\check{F}[k]=f_{k}$, where, for all $k \in$ $\mathbb{Z}^{d}, \quad f_{k}=\int_{\mathbb{T}^{d}} F(\gamma) e^{2 \pi i k \cdot \gamma} d \gamma$. There is a similar definition for $\check{\mu}$ where $\mu \in$ $M\left(\mathbb{T}^{d}\right)$. The support of a function $F$ is denoted by supp $F$. 


\subsection{Background}

As motivation, one would like to construct bounded sequences, $x: \mathbb{Z}^{d} \rightarrow \mathbb{C}$, whose autocorrelations are the inverse Fourier transforms of given positive measures. Problems of this kind arise in the construction of phase-coded waveforms, which in turn are particularly relevant in several critical, current applications in the areas of radar and communications see, e.g., [1], [5], [6], [16], [19], [20], [22], [23], [26], [27], [28].

In the setting of $\mathbb{R}$, we have the following theorem due to Wiener and Wintner [31], which was later extended to $\mathbb{R}^{d}$ in [2], [17].

Theorem 1.2. Let $\mu$ be a bounded positive Radon measure on $\mathbb{R}$. There is a constructible function $f \in L_{\text {loc }}^{\infty}(\mathbb{R})$ whose autocorrelation $A_{f}$ exists for all $t \in \mathbb{R}$, and $A_{f}=\check{\mu}$ on $\mathbb{R}$, i.e.,

$$
\forall t \in \mathbb{R}, \quad A_{f}(t)=\lim _{T \rightarrow \infty} \frac{1}{2 T} \int_{-T}^{T} f(t+s) \overline{f(s)} d s=\int_{\mathbb{R}} e^{2 \pi i t \gamma} d \mu(\gamma) .
$$

For any positive integer $N$, we denote the $d$-dimensional square in $\mathbb{Z}^{d}$ by $S(N)$, i.e.,

$$
S(N)=\left\{m=\left(m_{1}, m_{2}, \cdots, m_{d}\right) \in \mathbb{Z}^{d}:-N \leqslant m_{i} \leqslant N, i=1, \cdots, d\right\} .
$$

On $\mathbb{Z}^{d}$ the following version of the Wiener-Wintner theorem can be obtained [11].

Theorem 1.3. Let $F \in A\left(\mathbb{T}^{d}\right)$ be positive on $\mathbb{T}^{d}$. There is a constructible function $x: \mathbb{Z}^{d} \rightarrow \mathbb{C}$ such that

$$
\forall k \in \mathbb{Z}^{d}, \quad A_{x}[k]=\lim _{N \rightarrow \infty} \frac{1}{(2 N+1)^{d}} \sum_{m \in S(N)} x[k+m] \overline{x[m]}=\check{F}[k] .
$$

Although the Wiener-Wintner theorem gives the construction of the function $x$ it does not ensure boundedness of $x$. In fact, $x$ need not be an element of $\ell^{\infty}(\mathbb{Z})$ [18].

It has been shown in [29], [30] that if $\lambda \in(0,1)$ has binary expansion $0 . \alpha_{1} \alpha_{2} \alpha_{3} \cdots$, if we consider the Lebesgue measure on $(0,1)$, and if we define the unimodular (in fact, \pm 1 -valued) function $y$ by

$$
y[k]= \begin{cases}2 \alpha_{2 n+1}-1 & \text { if } k=n+1, n \in \mathbb{N} \cup\{0\} \\ 2 \alpha_{2 n}-1 & \text { if } k=1-n, n \in \mathbb{N},\end{cases}
$$

then, for almost all values of $\lambda$, the autocorrelation of $y, A_{y}$, is

$$
A_{y}[k]= \begin{cases}0 & \text { if } k \neq 0, \\ 1 & \text { if } k=0 .\end{cases}
$$

So $A_{y}$ is the inverse Fourier transform of $F \equiv 1$ on $\mathbb{T}$. Here Lebesgue measure on $(0,1)$ is the probability measure ([11], page 77$)$. 
Remark 1.4. A deterministic construction of a unimodular sequence on $\mathbb{Z}$ whose autocorrelation at integers is (1.2) is given in [29], where the sequence consists of $\pm 1 \mathrm{~s}$. An extensive generalization of this construction is given in [4].

\subsection{Outline}

Given $K>0$ and $M \in \mathbb{N}$, the function $\triangle_{K, M}(t)=K \max \left(1-\frac{|t|}{M}, 0\right)$ represents an isosceles triangle on the real line $\mathbb{R}$ having height $K$ and base $2 M$. In Section 2 , for a given $K$ and $M$, a constant amplitude sequence is constructed on the integers $\mathbb{Z}$ having autocorrelation $\triangle_{K, M}$ restricted to $\mathbb{Z}$ (Theorem 2.1). With this background, a large class of bounded sequences $z$ is shown to exist, where the autocorrelation of $z$ is a finite sum of such $\triangle_{K, M} \mathrm{~S}$ (Theorem 2.2). By taking an inverse Fourier transform we are led naturally to expansions in terms of Fejér functions. This line of thought is closely related to the construction of constant amplitude zero autocorrelation (CAZAC) sequences which, as referenced in Section 1.3, have applications in radar and communications.

In Section 3, we transition from $\mathbb{Z}$ to $\mathbb{R}$, first making some technical observations about algebraic bases for the space of even continuous piecewise linear functions on $\mathbb{R}$. Then, motivated by Theorem 2.2 , we prove Theorem 3.5 , which states that most such functions can be expanded as linear combinations of dilates of Fejér functions $\left(\frac{\sin \pi n \gamma}{\pi n \gamma}\right)^{2}$. Using the results of Section 3, in Section 4 we prove a multiplicative MRA decomposition formula (Theorem 4.2) and formulate a sampling theory in this setting (Example 4.3). In particular we prove that

$$
F(\gamma)=\sum_{m=0}^{\infty} \sum_{n=1}^{\infty} K_{m, n} \frac{n}{2^{m}}\left(\frac{\sin \pi \frac{n}{2^{m}} \gamma}{\pi \frac{n}{2^{m}} \gamma}\right)^{2} \quad \text { in } L^{2}(\widehat{\mathbb{R}})
$$

for a large class of even functions.

The dyadic formula (1.3) generalizes naturally and significantly. For each $m$, we consider the discrete group $G_{m}=\left\{\frac{n}{2^{m}}\right\}$ of $\mathbb{R}$, and these form an increasing sequence of groups as $m$ increases. The union is the group of all dyadic rationals. The fact that we only use positive $n$ in (1.3) is an artifact of dealing with even functions. In the limit we obtain the decomposition formula (1.3), but, for each $M$, when we sum from $m=0$ to $m=M$, we have an approximation of the given function, and this approximation has the zooming property which is a useful feature of classical dyadic MRA. We can obtain the same behavior for locally compact abelian groups $G$ having Sidon sets $\left\{g_{m}\right\}$ with which to generate the analogue of the $G_{m}$, where each $g_{m}$ corresponds to $\frac{1}{2^{m}}$ (see [24]). Further, we could retain the Fejér function on $G$, if we did not want to generalize further, by convolving step functions on $G$. 


\section{Sequences whose autocorrelation is the sum of tri- angles}

In this section we give some generalizations of (1.2), the autocorrelation function of (1.1) and of those sequences referred to in Remark 1.4. The results in this section, even though not directly used in later sections, provide a motivation for what we do there.

Theorem 2.1. Given $M \in \mathbb{N}$ and $K>0$. Let $A: \mathbb{Z} \rightarrow \mathbb{R}$ be defined by

$$
A[k]= \begin{cases}K\left(1-\frac{|k|}{M}\right) & \text { if } 0 \leqslant|k| \leqslant M, \\ 0 & \text { otherwise. }\end{cases}
$$

Then there exists a constructible sequence $x: \mathbb{Z} \rightarrow \mathbb{R}$ with constant amplitude $\sqrt{K}$ whose autocorrelation, $A_{x}$, is $A$.

Proof. As indicated in Remark 1.4 one can deterministically construct a unimodular sequence $y$ on $\mathbb{Z}$ whose autocorrelation is

$$
A_{y}[k]= \begin{cases}0 & \text { if } k \neq 0, \\ 1 & \text { if } k=0,\end{cases}
$$

and we use (2.2) at the end of the proof. Wiener's construction [29] of $y$ is as follows. On the positive integers let $y$ take values in the following order:

$[1,-1] \quad$ (this row has $1 \cdot 2^{1}$ elements and is repeated $2^{0}=1$ time);

$[1,1 ; 1,-1 ;-1,1 ;-1,-1]$ (this row has $2 \cdot 2^{2}$ elements and is repeated $2^{1}=2$ times);

$[1,1,1 ; 1,1,-1 ; 1,-1,1 ; 1,-1,-1 ;-1,1,1 ;-1,1,-1$;

$-1,-1,1 ;-1,-1,-1$ ] (this row has $3 \cdot 2^{3}$ elements and is repeated $2^{2}=4$ times); etc. Thus, $y[1]=1, y[2]=-1, y[3]=1, y[4]=1, \ldots$ In addition, let $y[0]=1$, and, for $k \in \mathbb{N}$, let $y[-k]=y[k]$.

We define the function $x: \mathbb{Z} \rightarrow \mathbb{C}$ by $x[k]=\sqrt{K} y\left[\left\lceil\frac{k}{M}\right\rceil\right]$, where $\lceil$.$\rceil denotes$ the next largest integer. Note that $|x|=\sqrt{K}$.

We show that the autocorrelation $A_{x}$ of $x$ is $A$ as defined in (2.1). Since $x$ is a real sequence, the autocorrelation function is even, and so it is enough to prove the result for $k>0$. Let $0 \leqslant M p \leqslant k \leqslant M(p+1)$ for some $p \in \mathbb{N} \cup\{0\}$. For any given integer $N$, let $n_{N}$ be the smallest integer such that $N<M\left(n_{N}+1\right)$. Then we have

$$
\begin{aligned}
A_{x}[k] & =\lim _{N \rightarrow \infty} \frac{1}{2 N+1} \sum_{m=-N}^{N} x[m+k] \overline{x[m]} \\
& =\lim _{N \rightarrow \infty} \frac{1}{2 N+1} \sum_{m=-M n_{N}}^{M n_{N}} x[k+m] x[m]+\lim _{N \rightarrow \infty} \frac{1}{2 N+1} \sum_{M n_{N}<|m| \leqslant N} x[m+k] x[m] \\
& =\lim _{N \rightarrow \infty}\left(S_{1, N}(k)+S_{2, N}(k)\right)=S_{1}(k)+S_{2}(k)
\end{aligned}
$$


First, we calculate bounds on $S_{2, N}(k)$.

$$
\begin{aligned}
\left|S_{2, N}(k)\right| & =\left|\frac{1}{2 N+1} \sum_{M n_{N}<|m| \leqslant N} x[m+k] x[m]\right| \\
& \leqslant \frac{1}{2 N+\frac{1}{M}} \sum_{n_{N}<|m| \leqslant N}|x[m+k] x[m]|=\frac{K}{2 N+1} \sum_{M n_{N}<|m| \leqslant N} 1=\frac{2 K\left(N-M n_{N}\right)}{2 N+1} .
\end{aligned}
$$

We know from the definition of $n_{N}$ that $N-M n_{N}<M$. Therefore, $S_{2}(k)=0$. Consequently, $A_{x}[k]=\lim _{N \rightarrow \infty} S_{1, N}(k)=S_{1}(k)$. Next, we write

$$
\begin{aligned}
S_{1, N}(k)= & \lim _{N \rightarrow \infty} \frac{1}{2 N+1} \sum_{m=-M n_{N}}^{M n_{N}} x[k+m] x[m] \\
= & \lim _{N \rightarrow \infty} \frac{1}{2 N+1} \sum_{n=-n_{N}}^{n_{N}-1} \sum_{m=M n+1}^{M(n+1)} x[k+m] x[m] \\
& \quad+\lim _{N \rightarrow \infty} \frac{1}{2 N+1} x\left[-n_{N}+k\right] x\left[-n_{N}\right] .
\end{aligned}
$$

Since $x$ has the same value $\sqrt{K} y[n+1]$ for all the integers $m \in[M n+1, M(n+1)]$, one can replace the $x[m]$ in the first term of the right side of $(2.4)$ by $\sqrt{K} y[n+1]$. Since the second term of the right side of (2.4) is 0 this implies

$$
\begin{aligned}
S_{1}(k)= & \lim _{N \rightarrow \infty} \frac{1}{2 N+1} \sum_{n=-n_{N}}^{n_{N}-1} \sum_{m=M n+1}^{M(n+1)} x[m+k] \sqrt{K} y[n+1] \\
= & \lim _{N \rightarrow \infty} \frac{1}{2 N+1}\left(\sum_{n=-n_{N}}^{n_{N}-1} \sum_{m=M n+1}^{M n+M(p+1)-k} x[m+k] \sqrt{K} y[n+1]\right. \\
& \left.+\sum_{n=-n_{N}}^{n_{N}-1} \sum_{M n+M(p+1)-k+1}^{M(n+1)} x[m+k] \sqrt{K} y[n+1]\right) \\
= & \lim _{N \rightarrow \infty} \frac{K}{2 N+1} \sum_{n=-n_{N}}^{n_{N}-1}\left(\sum_{m=M n+1}^{M n+M(p+1)-k} y[n+p+1] y[n+1]\right. \\
& \left.+\sum_{m=M n+M(p+1)-k+1} y[n+p+2] y[n+1]\right) \\
= & \lim _{N \rightarrow \infty} \frac{K}{2 N+1} \sum_{n=-n_{N}}^{n_{N}-1}((M(p+1)-k) y[n+p+1] y[n+1] \\
& +(k-M p) y[n+p+2] y[n+1])
\end{aligned}
$$




$$
\begin{aligned}
= & \lim _{N \rightarrow \infty} \frac{M(p+1)-k}{2 N+1} \frac{2 n_{N} K}{2 n_{N}} \sum_{n=-n_{N}}^{n_{N}-1} y[n+p+1] y[n+1]+ \\
& +\lim _{N \rightarrow \infty} \frac{(k-M p)}{2 N+1} \frac{2 n_{N} K}{2 n_{N}} \sum_{n=-n_{N}}^{n_{N}-1} y[n+p+2] y[n+1] .
\end{aligned}
$$

Since $n_{N} \rightarrow \infty$ as $N \rightarrow \infty$, we have

$$
\begin{aligned}
\lim _{N \rightarrow \infty} S_{1, N}(k)= & \lim _{N \rightarrow \infty} \frac{M(p+1)-k}{2 N+1} 2 n_{N} K A_{y}[p]+\lim _{N \rightarrow \infty} \frac{k-M p}{2 N+1} 2 n_{N} K A_{y}[p+1] \\
= & \lim _{N \rightarrow \infty}\left(p+1-\frac{k}{M}\right) \frac{2 n_{N} M}{2 N+1} K A_{y}[p] \\
& \quad+\lim _{N \rightarrow \infty}\left(\frac{k}{M}-p\right) \frac{2 n_{N} M}{2 N+1} K A_{y}[p+1] .
\end{aligned}
$$

Note that

$$
\lim _{N \rightarrow \infty} \frac{2 n_{N} M}{2 N+1}=1
$$

In fact, from the choice of $n_{N}$, we have $M n_{N} \leqslant N<M\left(n_{N}+1\right)$ so that $2 M n_{N}+1 \leqslant 2 N+1<2 M\left(n_{N}+1\right)+1$, and hence

$$
\frac{2 M n_{N}}{2 M\left(n_{N}+1\right)+1}<\frac{2 M n_{N}}{2 N+1} \leqslant \frac{2 M n_{N}}{2 M n_{N}+1} .
$$

$n_{N}$ goes to infinity as $N$ goes to infinity and so taking limits throughout as $N$ goes to infinity we obtain (2.6).

Substituting (2.6) in (2.5) and using the fact that $S_{2}(k)=0$, we obtain from (2.3) that

$$
A_{x}[k]=S_{1}(k)=K\left(p+1-\frac{k}{M}\right) A_{y}[p]+K\left(\frac{k}{M}-p\right) A_{y}[p+1] .
$$

If $0 \leqslant k \leqslant M$ then $p=0$. For every other range of $k, p$ is non-zero. Using the values of $A_{y}[p]$ as given by $(2.2)$ and the fact that $A_{x}$ is an even function one obtains (2.1).

Theorem 2.2. (a) Let $X$ be the set of constant amplitude functions $x: \mathbb{Z} \rightarrow \mathbb{C}$ such that for some positive integer $M$

$$
A_{x}[k]= \begin{cases}1-\frac{|k|}{M}, & \text { if } 0 \leqslant|k| \leqslant M, \\ 0, & \text { otherwise. }\end{cases}
$$

Then there is a well defined finite Borel measure $p$ on $X$ induced from Lebesgue measure on $(0,1)$, in a manner described in the proof.

(b) For almost all $x, y \in X$, with respect to $p$, we have

$$
A_{x+y}=A_{x}+A_{y},
$$


noting that $x+y$ does not necessarily have constant amplitude and that $A_{x+y}$ is not generally a triangle.

Proof. (a) Using the formulation of (1.1) and (1.2), as well as Theorem 2.1, we complete the construction of $p$ below in the process of proving part (b).

(b) We have already seen the construction of such $x$ and $y$ in Theorem 2.1. Formally,

$$
\begin{aligned}
A_{x+y}[k]= & \lim _{N \rightarrow \infty} \frac{1}{2 N+1} \sum_{m=-N}^{N}(x+y)[m+k] \overline{(x+y)[m]} \\
= & \lim _{N \rightarrow \infty} \frac{1}{2 N+1} \sum_{m=-N}^{N}(x[m+k]+y[m+k])(\overline{x[m]}+\overline{y[m]}) \\
= & \lim _{N \rightarrow \infty} \frac{1}{2 N+1} \sum_{m=-N}^{N} x[m+k] \overline{x[m]}+\lim _{N \rightarrow \infty} \frac{1}{2 N+1} \sum_{m=-N}^{N} y[m+k] \overline{y[m]}+ \\
& +\lim _{N \rightarrow \infty} \frac{1}{2 N+1} \sum_{m=-N}^{N} x[m+k] \overline{y[m]}+\lim _{N \rightarrow \infty} \frac{1}{2 N+1} \sum_{m=-N}^{N} y[m+k] \overline{x[m]} \\
= & A_{x}(k)+A_{y}(k)+\lim _{N \rightarrow \infty} \frac{1}{2 N+1} \sum_{m=-N}^{N} x[m+k] \overline{y[m]}+ \\
& +\lim _{N \rightarrow \infty} \frac{1}{2 N+1} \sum_{m=-N}^{N} y[m+k] \overline{x[m]} .
\end{aligned}
$$

Let us denote the last two terms on the right side of (2.7) by $S_{3}$ and $S_{4}$, respectively. We want to show that $S_{3}=0$ and $S_{4}=0$.

$$
S_{3}=\lim _{N \rightarrow \infty} \frac{1}{2 N+1} \sum_{m=-N}^{N} x[m+k] \overline{y[m]} .
$$

Without loss of generality we take $y$ to be real-valued and so (2.8) becomes

$$
S_{3}=\lim _{N \rightarrow \infty} \frac{1}{2 N+1} \sum_{m=-N}^{N} x[m+k] y[m] .
$$

Suppose that

$$
A_{x}[k]= \begin{cases}1-\frac{|k|}{M_{1}}, & \text { if } 0 \leqslant|k| \leqslant M_{1}, \\ 0, & \text { otherwise, }\end{cases}
$$

and

$$
A_{y}[k]= \begin{cases}1-\frac{|k|}{M_{2}}, & \text { if } 0 \leqslant|k| \leqslant M_{2}, \\ 0, & \text { otherwise. }\end{cases}
$$


Let $P_{N}$ be the largest integer so that

$$
M_{2} P_{N} \leqslant N \leqslant M_{2}\left(P_{N}+1\right) .
$$

Then $S_{3}$ can be written as

$$
\begin{aligned}
S_{3}= & \lim _{N \rightarrow \infty} \frac{1}{2 N+1} \sum_{m=-N}^{-M_{2} P_{N}-1} x[m+k] y[m]+\lim _{N \rightarrow \infty} \frac{1}{2 N+1} \sum_{m=M_{2} P_{N}+1}^{N} x[m+k] y[m]+ \\
& +\lim _{N \rightarrow \infty} \frac{1}{2 N+1} \sum_{m=-M_{2} P_{N}}^{M_{2} P_{N}} x[m+k] y[m] .
\end{aligned}
$$

Let us denote the first two terms of (2.11) by $s_{1}$ and $s_{2}$, respectively. Now,

$$
\left|s_{1}\right| \leqslant \sum_{m=-N}^{-M_{2} P_{N}-1} 1=N-M_{2} P_{N}
$$

and

$$
\left|s_{2}\right| \leqslant \sum_{m=M_{2} P_{N}+1}^{N} 1=N-M_{2} P_{N} .
$$

From (2.10),

$$
N-M_{2} P_{N} \leqslant M_{2}\left(P_{N}+1\right)-M_{2} P_{N}=M_{2}
$$

which means $\left|s_{1}\right| \leqslant M_{2}$ and $\left|s_{2}\right| \leqslant M_{2}$. Therefore,

$$
\lim _{N \rightarrow \infty} \frac{\left|s_{1}\right|}{2 N+1} \leqslant \lim _{N \rightarrow \infty} \frac{M_{2}}{2 N+1}=0
$$

and also

$$
\lim _{N \rightarrow \infty} \frac{\left|s_{2}\right|}{2 N+1} \leqslant \lim _{N \rightarrow \infty} \frac{M_{2}}{2 N+1}=0
$$

Thus,

$$
\begin{aligned}
S_{3}= & \lim _{N \rightarrow \infty} \frac{1}{2 N+1} \sum_{m=-M_{2} P_{N}}^{M_{2} P_{N}} x[m+k] y[m] \\
= & \lim _{N \rightarrow \infty} \frac{1}{2 N+1} \sum_{n=-P_{N}}^{P_{N}-1} \sum_{m=M_{2} n+1}^{M_{2}(n+1)} x[m+k] y[m]+ \\
& +\lim _{N \rightarrow \infty} \frac{1}{2 N+1} x\left[-M_{2} P_{N}+k\right] y\left[-M_{2} P_{N}\right] \\
= & \lim _{N \rightarrow \infty} \frac{1}{2 N+1} \sum_{n=-P_{N}}^{P_{N}-1} \sum_{m=M_{2} n+1}^{M_{2}(n+1)} x[m+k] y\left[M_{2}(n+1)\right] .
\end{aligned}
$$


The last step (2.13) follows due to the fact that by construction $y$ is constant and equal to either +1 or -1 in the interval $\left[M_{2} n+1, M_{2}(n+1)\right]$. So $y\left[M_{2}(n+1)\right]$ is either +1 or -1 . Between $\left(M_{2} n+1\right)$ and $M_{2}(n+1)$ there are $M_{2}$ terms. So there are $M_{2}$ values of $x$. Suppose that of these $M_{2}$ values there are $j$ that have the value +1 and $\left(M_{2}-j\right)$ that have the value -1 . Upon multiplication by $y\left(M_{2}(n+1)\right)$ we have either $j$ values that are -1 and $\left(M_{2}-j\right)$ values that are +1 or vice versa. In the sum on the right side of (2.13) there are $2 P_{N}$ blocks of length $M_{2}$. Let us say that the first block has $j_{1}$ terms equal to +1 and $\left(M_{2}-j_{1}\right)$ terms equal to -1 , the second block has $j_{2}$ terms equal to +1 and $\left(M_{2}-j_{2}\right)$ terms equal to -1 and so on. Together, there are $\left(j_{1}+j_{2}+\cdots+j_{2 P_{N}}\right)$ terms equal to +1 and $\left(M_{2}-j_{1}+M_{2}-j_{2}+\cdots+M_{2}-j_{2 P_{N}}\right)=2 P_{N} M_{2}-\left(j_{1}+j_{2}+\cdots+j_{2 P_{N}}\right)$ terms equal to -1 . Let $P_{N} M_{2}=M$ and $j_{1}+j_{2}+\cdots+j_{2 P_{N}}=M-j$ where $-M \leqslant j \leqslant M$. Note that this $M$ is unrelated to the $M$ that appears in part (a) of the statement of this theorem. Then $2 P_{N} M_{2}-\left(j_{1}+j_{2}+\cdots+j_{2 P_{N}}\right)=2 M-(M-j)=M+j$. Thus, out of $2 M$ consecutive values of $x[m+k] y[m]$ there are $(M-j)$ values that are +1 and $(M+j)$ values that are -1 . So the absolute value of the sum of $2 P_{N} M_{2}$ consecutive values of $x[m+k] y[m]$ would be $M+j-(M-j)=2|j|$. Hence, the sum of these values exceeds $M \epsilon$ in absolute value if $[M \epsilon] \leqslant 2|j| \leqslant 2 M$. The number of ways of having $(M-j)+1 \mathrm{~s}$ and $(M+j)-1 \mathrm{~s}$ is $\left(\begin{array}{c}2 M \\ M-j\end{array}\right)=\left(\begin{array}{c}2 M \\ M+j\end{array}\right)$.

Let $\Omega$ be the set of all possibilities for the $2 M$ consecutive values of $x[m+$ $k] y[m]$. From (1.1), each such $x$ and $y$ corresponds to some $\lambda \in(0,1)$. For a subset $E$ of $\Omega$ we define a measure on $\Omega$ as $p(E)=\frac{|E|}{|\Omega|}$. The total number of possible values of the $2 M$ consecutive values of $x[m+k] y[m]$ is $2^{2 M}$ and so $|\Omega|=2^{2 M}$. From our discussion in the previous paragraph it follows that given $\epsilon$ the measure of the set for which the sum of $2 M$ consecutive values exceeds $M \epsilon$ in absolute value is

$$
2^{-2 M} \sum_{|j|=\left[\frac{M \epsilon}{2}\right]}^{M}\left(\begin{array}{c}
2 M \\
M-j
\end{array}\right)=2^{-2 M+1} \sum_{j=\left[\frac{M \epsilon}{2}\right]}^{M}\left(\begin{array}{c}
2 M \\
M-j
\end{array}\right) .
$$

It can be shown in a manner identical to that in $[29]$ that

$$
\lim _{M \rightarrow \infty} 2^{-2 M+1} \sum_{j=\left[\frac{M \epsilon}{2}\right]}^{M}\left(\begin{array}{c}
2 M \\
M-j
\end{array}\right)=0 .
$$

Thus the set of $x$ and $y$ for which there should fail to be an integral value of $M=P_{N} M_{2}$ such that from that value on (see (2.12))

$$
\left|\sum_{m=-M}^{M} x[m+k] y[m]\right| \leqslant M \epsilon+1
$$


has measure zero. Therefore,

$$
\varlimsup_{N \rightarrow \infty}\left|\frac{1}{2 N+1} \sum_{m=-M}^{M} x[m+k] y[m]\right| \leqslant \frac{M \epsilon+1}{2 N+1}=\frac{P_{N} M_{2} \epsilon}{2 N+1}+\frac{1}{2 N+1} .
$$

From (2.10),

$$
\frac{P_{N} M_{2}}{2 N+1} \leqslant \frac{N}{2 N+1} \rightarrow \frac{1}{2}
$$

as $N$ goes to infinity. So, the left side of (2.14) is less than $\frac{\epsilon}{2}$ and for almost all $x$ and $y$,

$$
\lim _{N \rightarrow \infty} \frac{1}{2 N+1} \sum_{m=-N}^{N} x[m+k] y[m]=0 .
$$

In a similar way one can show that

$$
S_{4}=\lim _{N \rightarrow \infty} \frac{1}{2 N+1} \sum_{m=-N}^{N} y[m+k] x[m]=0
$$

for almost every $\lambda$ and hence for almost every $x$ and $y$. This concludes proving part (b).

Example 2.3. Generally, $A_{x+y}[k] \neq A_{x}[k]+A_{y}[k]$. In fact, in the case of realvalued sequences $x, y \in \ell^{\infty}(\mathbb{Z})$, when all limits as $N \rightarrow \infty$ exist, $A_{x+y}[k]=$ $A_{x}[k]+A_{y}[k]+2 A_{x y}[-k]$, and there is no reason to expect $A_{x y}[-k]=0$ for each $k \in \mathbb{Z}$. Here, $A_{x y}$ is the cross-correlation of $x$ and $y$ defined by

$$
\forall k \in \mathbb{Z}, A_{x y}[k]=\lim _{N \rightarrow \infty} \frac{1}{2 N+1} \sum_{m=-N}^{N} x[k+m] \overline{y[m]} .
$$

As a particular example, note that the binary expansions, with a precision of 16 bit, of $\lambda_{x}=0.35$ and $\lambda_{y}=0.9$ are 0.01011001100110011 and 0.1110011001100110 , respectively. From these one can obtain sequences $x$ and $y$ of \pm 1 s by following the definition of $y$ in (1.1). The partial autocorrelations of $x, y$, and $x+y$ have been calculated by computing the sum in Definition 1.1 for $N=1000$, i.e., $2 N+1=2001$ terms. These partial autocorrelations at the integers between -10 and 10 are plotted in Figure 1. Clearly, the sums of the autocorrelations of $x$ and $y$ do not match the autocorrelation of $x+y$.

\section{A decomposition formula in terms of Fejér func- tions}

Moving from the setting of $\mathbb{Z}$ to the setting of $\mathbb{R}$, we shall now establish Theorem 3.5 which states that a certain class of even functions can be written as 

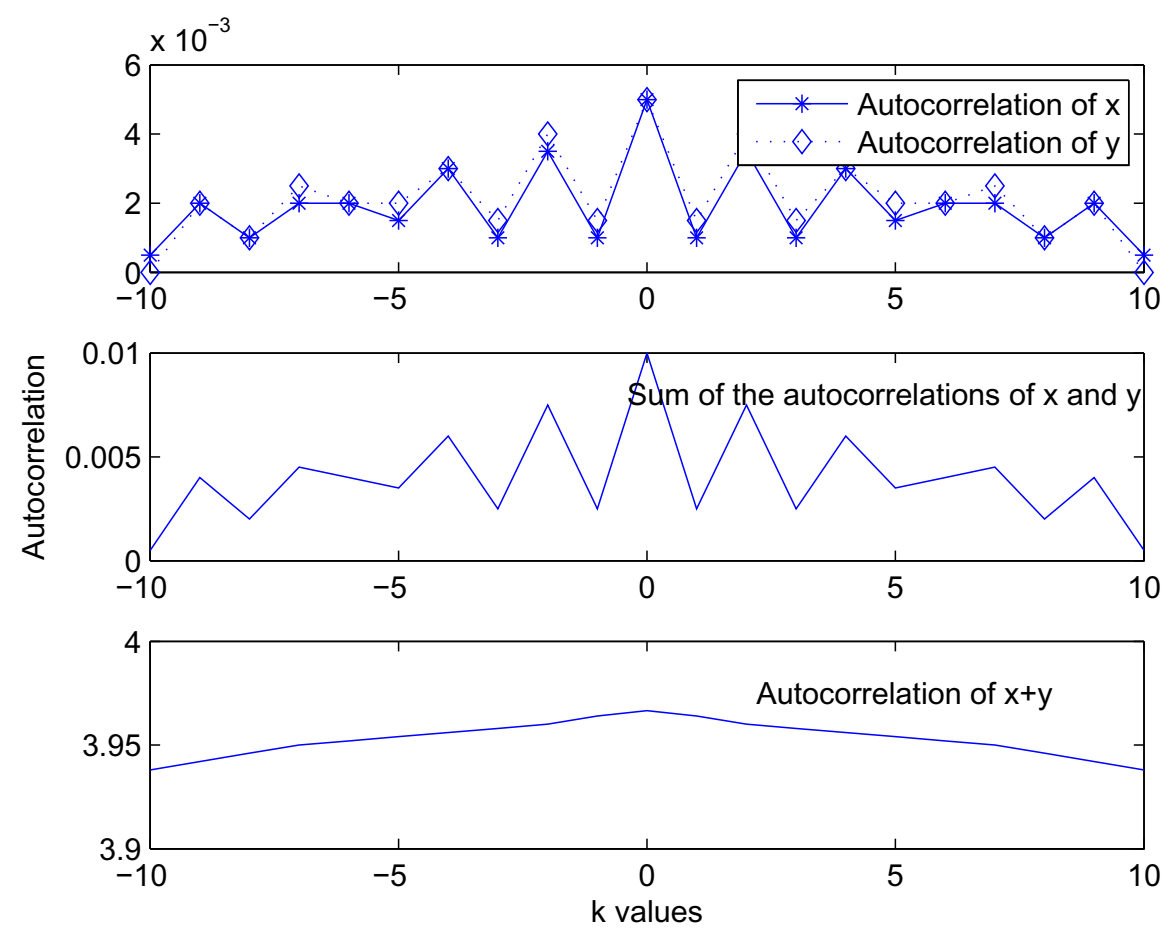

Figure 1: Autocorrelations of two sequences $x$ and $y$ and their sum

a linear combination of Fejér functions $\left(\frac{\sin \pi n \gamma}{\pi n \gamma}\right)^{2}$. By what has been done in Section 2 , in the setting of $\mathbb{Z}$, this implies that bounded real valued sequences can be constructed whose autocorrelations are inverse Fourier transforms of linear combinations of such functions, see Example 3.6. By taking inverse Fourier transforms, Theorem 3.5 as well as Theorem 4.2 in the next section can be formulated in the context of spline decompositions, e.g., see [13] and the comment before Theorem 4.2 .

Let $\left\{h_{k}\right\}_{k \in \mathbb{Z}}$ be the family of continuous triangle hat functions on $\mathbb{R}$ defined by

$$
h_{k}(x)= \begin{cases}1 & \text { when } x=k \\ \text { linear } & \text { on }[k, k+1] \text { and }[k-1, k] \\ 0 & \text { otherwise }\end{cases}
$$

Lemma 3.1. (a) Let $X$ be the complex vector space of continuous functions on $\mathbb{R}$ that are piecewise linear on the intervals $[k, k+1]_{k \in \mathbb{Z}}$. The sequence $\left\{h_{k}\right\}_{k \in \mathbb{Z}}$ of hat functions is an algebraic basis for $X$ in the sense that each $f \in X$ has the unique representation $f=\sum_{k} f(k) h_{k}$ meaning that

$$
\forall C \subseteq \mathbb{R}, \text { compact, } \exists N_{C} \text { such that } \forall N \geq N_{C}, f=\sum_{|k| \leq N} f(k) h_{k} \text { on } C .
$$


(b) The family $h_{0} \cup\left\{h_{k}+h_{-k}\right\}_{k=1}^{\infty}$ forms a basis for all even continuous functions on $\mathbb{R}$ that are piecewise linear on the intervals $\left.[k, k+1]\right|_{k \in \mathbb{Z}}$.

Proof. (a) The $h_{k}$ s are linearly independent. In fact, assume $H=\sum_{k} c_{k} h_{k}=0$ and evaluate $H$ at $j \in \mathbb{Z}$. Since only $h_{j}$ is non-zero at $j$, and is equal to 1 there, we have $H(j)=c_{j}=0$ for all $j$.

Let $f$ be continuous on $\mathbb{R}$ and piecewise linear on $\left.[k, k+1]\right|_{k \in \mathbb{Z}}$. By the following argument, we see that $f=\sum_{k} f(k) h_{k}$ which proves that the $h_{k}$ s also form a spanning set. Let

$$
e=f-\sum_{k} f(k) h_{k}
$$

Then $e(j)=0$ for all $j$ since $h_{j}(k)=\delta_{j k}$. Also, $e$ restricted to $[k, k+1]_{k \in \mathbb{Z}}$ is linear and $e(k)=e(k+1)=0$ which implies that $e=0$ on $[k, k+1]_{k \in \mathbb{Z}}$. In particular,

$\sum_{k} f(k) h_{k}(x)=x(f(k+1)-f(k))+(f(k)(k+1)-k f(k+1)) \quad$ on $\quad[k, k+1]$.

Thus $f=\sum_{k} f(k) h_{k}$ and the $h_{k}$ s form a spanning set.

(b) Let $f$ be an even continuous function on $\mathbb{R}$ that is piecewise linear on the intervals $\left.[k, k+1]\right|_{k \in \mathbb{Z}}$. By (a),

$$
f=\sum_{k} c_{k} h_{k}
$$

Since $h_{j}(k)=\delta_{j k}$, we have

$$
f(k)=c_{k} .
$$

Again since $f$ is even,

$$
f(k)=f(-k)=c_{-k},
$$

and so

$$
f=f(0) h_{0}+\sum_{k=1}^{\infty} f(k)\left(h_{k}+h_{-k}\right) .
$$

This implies that $h_{0} \cup\left\{h_{k}+h_{-k}\right\}_{k=1}^{\infty}$ spans that space of even continuous functions on $\mathbb{R}$ that are piecewise linear on the intervals $\left.[k, k+1]\right|_{k \in \mathbb{Z}}$. That this set is also linearly independent can shown in a manner identical to part (a). One can thus conclude that the family $h_{0} \cup\left\{h_{k}+h_{-k}\right\}_{k=1}^{\infty}$ forms a basis for even continuous functions on $\mathbb{R}$ that are piecewise linear on the intervals $\left.[k, k+1]\right|_{k \in \mathbb{Z}}$.

Remark 3.2. The previous discussion on algebraic basis raises the question about Riesz basis properties of these hat functions. By Lemma 3.6.10 and Theorem 3.6.6 in [9], $\left\{h_{k}\right\}_{k \in \mathbb{Z}}$ is a Riesz basis for $\overline{\operatorname{span}}\left\{h_{k}\right\}_{k \in \mathbb{Z}} \subseteq L^{2}(\mathbb{R})$. 
Let $\triangle_{n}=\triangle_{1, n}$, i.e.,

$$
\triangle_{n}(t)=\max \left(1-\frac{|t|}{n}, 0\right) .
$$

Lemma 3.3. The triangle $\triangle_{n}$ can be expressed as a linear combination of the first $n$ hats, i.e.,

$$
\triangle_{n}(t)=c_{0} h_{0}+\sum_{k=1}^{n-1} c_{k}\left(h_{k}(t)+h_{-k}(t)\right) \quad \text { for } n \geq 2, \quad \text { and } \quad \triangle_{1}=h_{0} .
$$

Proof. By definition, $\triangle_{1}=h_{0}$. For each $n, \triangle_{n}$ is an even continuous function that is piecewise linear on the intervals $\left.[k, k+1]\right|_{k \in \mathbb{Z}}$. From (3.2) we know that

$$
\triangle_{n}=\triangle_{n}(0) h_{0}+\sum_{k=1}^{\infty} \triangle_{n}(k)\left(h_{k}+h_{-k}\right)
$$

Since the supp $\triangle_{n}=[-n, n]$ and $\triangle_{n}(n)=\triangle_{n}(-n)=0$,

$$
\triangle_{n}=\triangle_{n}(0) h_{0}+\sum_{k=1}^{n-1} \triangle_{n}(k)\left(h_{k}+h_{-k}\right)
$$

Also, $\triangle_{n}(0)=1$ and $\triangle_{n}(k)=\frac{n-k}{n}$ for $k \in \mathbb{Z}, 0<|k|<n$. Thus

$$
\triangle_{n}=h_{0}+\sum_{k=1}^{n-1} \frac{n-k}{n}\left(h_{k}+h_{-k}\right) \text {. }
$$

Proposition 3.4. Let $f$ be an even continuous function on $\mathbb{R}$ that is piecewise linear on the intervals $[k, k+1]_{k \in \mathbb{Z}}$. Then $f$ has a unique representation as a linear combination of the triangles $\triangle_{n}$ with pointwise convergence on $\mathbb{R}$.

Proof. Let $H_{1}=h_{0}$ and for $k \neq 0$ let $H_{k+1}=h_{k}+h_{-k}$. Then by (3.3) of Lemma 3.3 we have

$$
\left[\begin{array}{c}
\triangle_{1} \\
\triangle_{2} \\
\vdots \\
\triangle_{n}
\end{array}\right]=\left[\begin{array}{ccccc}
1 & 0 & 0 & \cdots & 0 \\
1 & \frac{1}{2} & 0 & \cdots & 0 \\
\vdots & \vdots & \vdots & \ddots & \\
1 & \cdots & & \frac{2}{n} & \frac{1}{n}
\end{array}\right]\left[\begin{array}{c}
H_{1} \\
H_{2} \\
\vdots \\
H_{n}
\end{array}\right]
$$

or, $T=A H$, 
where $A$ is lower triangular with positive diagonal entries, and thus can be inverted.

$$
A^{-1}=\left[\begin{array}{rrrrrr}
1 & 0 & 0 & 0 & \cdots & 0 \\
-2 & 2 & 0 & 0 & \cdots & 0 \\
1 & -4 & 3 & 0 & \cdots & 0 \\
0 & 2 & -6 & 4 & \cdots & 0 \\
\vdots & \vdots & \ddots & \ddots & \ddots & \vdots \\
0 & 0 & \cdots & (n-2) & -2(n-1) & n
\end{array}\right] .
$$

Each $H_{k}$ can be written uniquely as a linear combination of the first $k$ triangles, i.e.,

$$
\begin{aligned}
H_{1} & =\triangle_{1} ; \\
H_{2} & =-2 \triangle_{1}+2 \triangle_{2} ; \\
H_{3} & =\triangle_{1}-4 \triangle_{2}+3 \triangle_{3} ; \\
H_{4} & =2 \triangle_{2}-6 \triangle_{3}+4 \triangle_{4} ; \\
\vdots & \\
H_{n} & =(n-2) \triangle_{n-2}-2(n-1) \triangle_{n-1}+n \triangle_{n} .
\end{aligned}
$$

As shown in Lemma 3.1, the $H_{k}$ s form a basis for the space of even continuous

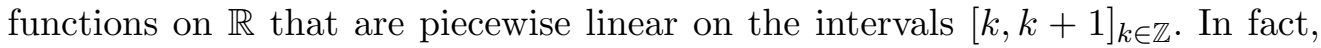
(3.2) can be written as

$$
f=\sum_{k=1}^{\infty} f(k-1) H_{k}
$$

In terms of the triangles this becomes

$$
\begin{aligned}
f=f & (0) \triangle_{1}+f(1)\left(-2 \triangle_{1}+2 \triangle_{2}\right)+f(2)\left(\triangle_{1}-4 \triangle_{2}+3 \triangle_{3}\right)+ \\
& f(3)\left(2 \triangle_{2}-6 \triangle_{3}+4 \triangle_{4}\right)+\cdots+ \\
& f(k-1)\left((k-2) \triangle_{k-2}-2(k-1) \triangle_{k-1}+k \triangle_{k}\right)+ \\
& f(k)\left((k-1) \triangle_{k-1}-2 k \triangle_{k}+(k+1) \triangle_{k+1}\right)+ \\
& f(k+1)\left(k \triangle_{k}-2(k+1) \triangle_{k+1}+(k+2) \triangle_{k+2}\right)+\cdots \\
=( & f(0)-2 f(1)+f(2)) \triangle_{1}+(2 f(1)-4 f(2)+2 f(3)) \triangle_{2}+ \\
& (3 f(2)-6 f(3)+3 f(4)) \triangle_{3}+\cdots+ \\
& (k f(k-1)-2 k f(k)+k f(k+1)) \triangle_{k}+\cdots .
\end{aligned}
$$

This gives the result.

In the following we choose $F \in L^{1}(\widehat{\mathbb{R}}) \cap L^{2}(\widehat{\mathbb{R}}) \cap A(\widehat{\mathbb{R}}): L^{1}$, since we are interested in point values of $\check{F} ; L^{2}$, since we are making an assertion about $L^{2}$ convergence whose norm preserves Euclidean distance between finite energy 
signals and their spectral representations; $A$, because of the convergence hypothesis in Theorem 3.5 and its relation to quadrature formulas. Of course, $L^{1}, L^{2}$, and $A$ are natural settings for a great deal of harmonic analysis since $\widehat{L^{1}}=A$, $L^{2} * L^{2}=A$, and an intrinsic characterization of $A(\widehat{\mathbb{R}})$ has proven so elusive. Further, $F \in L^{1} \cap L^{2} \cap A$ is equivalent to saying that both $F$ and $\check{F}$ are in the Segal algebra $L^{1} \cap L^{2}$.

Theorem 3.5. Let $F \in L^{1}(\widehat{\mathbb{R}}) \cap L^{2}(\widehat{\mathbb{R}}) \cap A(\widehat{\mathbb{R}})$ be an even function whose inverse Fourier transform $f=\check{F}$ is piecewise linear on the intervals $[k, k+1]_{k \in \mathbb{Z}}$ and continuous on $\mathbb{R}\left(\right.$ by $\left.F \in L^{1}(\widehat{\mathbb{R}})\right)$. Assume

$$
\sum_{n=1}^{\infty} n^{3 / 2}|f(n)|<\infty
$$

Then $F$ can be written as a linear combination of Fejér functions, i.e.,

$$
F(\gamma)=\sum_{n=1}^{\infty} n K_{n}\left(\frac{\sin \pi n \gamma}{\pi n \gamma}\right)^{2}
$$

where the convergence is in the $L^{2}(\widehat{\mathbb{R}})$ norm.

Proof. The inverse Fourier transform $f$ of $F$ satisfies the hypotheses of Proposition 3.4 and so

$$
f(t)=\sum_{n=1}^{\infty} K_{n} \max \left(1-\frac{|t|}{n}, 0\right),
$$

with pointwise convergence on $\mathbb{R}$, where each

$$
K_{n}=n(f(n-1)-2 f(n)+f(n+1)),
$$

because of Proposition 3.4. Since $F \in L^{1}(\widehat{\mathbb{R}})$ we see that $\sum_{n=1}^{\infty} K_{n}=f(0)$.

The convergence assertion follows from Plancherel's theorem and the following estimate using Minkowski's inequality.

$$
\begin{aligned}
& \left(\int_{\mathbb{R}}\left|\sum_{n=N+1}^{\infty} K_{n} \max \left(1-\frac{|t|}{n}, 0\right)\right|^{2} d t\right)^{1 / 2} \\
& \leqslant \sum_{n=N+1}^{\infty}\left(\int_{\mathbb{R}}\left|K_{n} \max \left(1-\frac{|t|}{n}, 0\right)\right|^{2}\right)^{1 / 2} \\
& =\sqrt{2} \sum_{n=N+1}^{\infty}\left|K_{n}\right|\left(\int_{0}^{n}\left(1-\frac{t}{n}\right)^{2} d t\right)^{1 / 2} \\
& =\sqrt{\frac{2}{3}} \sum_{n=N+1}^{\infty} \sqrt{n}\left|K_{n}\right|
\end{aligned}
$$


The right side is finite and goes to 0 as $N$ goes to infinity by the formulas for $K_{n}$ in terms of $f(n)$ and by our assumption.

Minkowski's inequality is not refined; and weaker but uglier assumptions can be made to ensure the $L^{2}$-convergence asserted in Theorem 3.5.

Example 3.6. In the setting of $\mathbb{Z}$, suppose $F: \mathbb{T} \rightarrow \mathbb{C}$. Its inverse Fourier transform is defined on $\mathbb{Z}$, i.e., $\check{F}: \mathbb{Z} \rightarrow \mathbb{C}$. Suppose $\breve{F}$ vanishes except for a finite subset of $\mathbb{Z}$. We assume that if we extend $\check{F}$ continuously to $\mathbb{R}$ by defining its graph linearly between each pair $(k, \check{F}(k))$ and $(k+1, \check{F}(k+1))$, then $\check{F}$ has the same properties as Theorem 3.5. Then one can construct a sequence $x: \mathbb{Z} \rightarrow \mathbb{C}$ such that $x \in \ell^{\infty}(\mathbb{Z})$ and the autocorrelation of $x$ is $\check{F}$, the inverse Fourier transform of $F(\gamma)=\sum_{n=1}^{N} n K_{n}\left(\frac{\sin \pi n \gamma}{\pi n \gamma}\right)^{2}$. This is due to the following.

On $\mathbb{Z}$, under the assumptions stated,

$$
\check{F}[m]=\sum_{n=1}^{N} K_{n} \max \left(1-\frac{|m|}{n}, 0\right)=\sum_{n=1}^{N} K_{n} \triangle_{n}[m] .
$$

Each triangle $K_{n} \triangle_{n}$ is the autocorrelation of a sequence $x_{n}$ of the form discussed in Section 2 with $\left|x_{n}\right|=\sqrt{K_{n}}$. Due to what has been discussed in Section 2 the sequence $x$ whose autocorrelation is $\check{F}$ would be $x=x_{1}+\cdots+x_{N}$ and $|x|$ is bounded by $\sum_{n=1}^{N} \sqrt{K_{n}}$.

Remark 3.7. The functions $h_{k}, k \neq 0$, as defined in (3.1), are translations of the function $h_{0}$. On the other hand, the triangles $\triangle_{k}, k \neq 1$, are dilations of the triangle $\triangle_{1}$. In view of the transformation given by (3.4), this implies that the translations of the $h_{k} \mathrm{~s}$ are transformed into dilations of the $\triangle_{k} \mathrm{~s}$.

Remark 3.8. For perspective on the representation formula (3.5), the Classical Sampling Theorem asserts that any bandlimited function $f$ with supp $\hat{f} \subseteq$ $[-\Omega, \Omega]$ and sampling period $T$ with $0<T \leq \frac{1}{2 \Omega}$ can be expressed in terms of its values sampled at integer multiples of $T$. Mathematically, in the case $2 T \Omega=1$,

$$
f(t)=T \sum_{n} f(n T) \frac{\sin 2 \pi \Omega(t-n T)}{\pi(t-n T)}
$$

with convergence in $L^{2}(\mathbb{R})$ and uniformly on $\mathbb{R}$. A disadvantage with (3.6) is that it can give rise to the Gibbs phenomenon at points of discontinuity if $f$ is piecewise continuous. One way to avoid the Gibbs phenomenon is to use certain positive functions and the Fejér kernel as used in (3.5) is a good choice for this purpose. Several sampling results in terms of the Fejér function can be found in [8], [14], [15], and [21]. In addition to eliminating Gibbs, Theorem 3.5 can be thought of as generalizing the Classical Sampling Theorem to a class of functions that are not bandlimited, i.e., functions $f$ with supp $\hat{f}$ not compact. We should 
point out that in the case $2 T \Omega<1$ sampling functions having smooth Fourier transforms can be used to great advantage, see [3], Chapter 3.10. A discussion on aliasing and non-bandlimited functions in the Classical Sampling Theorem can be found in pages $445-507$ of the edited volume [10], cf. [7].

\section{Relation to multiresolution analysis}

One can generalize the situation of Section 3 by extending Proposition 3.4 and Theorem 3.5 to the class of even functions $F \in A(\widehat{\mathbb{R}})$ whose inverse Fourier transforms $\check{F}$ are piecewise linear on the intervals $\left.\left[2^{-m} n, 2^{-m}(n+1)\right]\right|_{n \in \mathbb{Z}}, m \in \mathbb{N} \cup\{0\}$, and continuous on $\mathbb{R}$. This would incorporate another operation corresponding to obtaining finer and finer scales, hence giving an analogue of multiresolution analysis. This is what is done in the present section.

Definition 4.1. For each $m \in \mathbb{N} \cup\{0\}$ one can define the continuous triangle hat functions and triangles with respect to the intervals $\left.\left[\frac{n}{2^{m}}, \frac{n+1}{2^{m}}\right]\right|_{n \in \mathbb{Z}}$ by

$$
h_{n}^{(m)}(t)=\left\{\begin{array}{cc}
1 & \text { if } t=2^{-m} n \\
\text { linear } & \text { in }\left[2^{-m}(n-1), 2^{-m} n\right] \text { and }\left[2^{-m} n, 2^{-m}(n+1)\right] \\
0 & \text { otherwise }
\end{array}\right.
$$

and

$$
\triangle_{n}^{(m)}(t)=\max \left(1-\frac{2^{m}|t|}{n}, 0\right)
$$

respectively. The $h_{n}^{(m)}$ s form an algebraic basis for the space of continuous functions on $\mathbb{R}$ that are piecewise linear on the intervals $\left.\left[2^{-m} n, 2^{-m}(n+1)\right]\right|_{n \in \mathbb{Z}}$. Lemma 3.3 can be proved in the same way for the $h_{n}^{(m)} \mathrm{s}$ and $\triangle_{n}^{(m)} \mathrm{s}$. Thus the $\triangle_{n}^{(m)}$ S also form a basis for the space of continuous functions on $\mathbb{R}$ that are piecewise linear on the intervals $\left.\left[2^{-m} n, 2^{-m}(n+1)\right]\right|_{n \in \mathbb{Z}}$.

Let $V_{m}$ be the space of continuous functions on $\mathbb{R}$ that are piecewise linear on the intervals $\left[2^{-m} n, 2^{-m}(n+1)\right]_{n \in \mathbb{Z}}$, i.e.,

$$
V_{m}=\left\{\text { Continuous } f \in L^{2}(\mathbb{R}):\left.f\right|_{\left[2^{-m} n, 2^{-m}(n+1)\right]} \text { is linear }\right\} .
$$

In particular, each $V_{m} \subseteq V_{m+1}$. In fact, these spaces form a multiresolution analysis of $L^{2}(\mathbb{R})$, whose scaling function is the piecewise linear spline

$$
\phi(t)= \begin{cases}1-|t| & 0 \leqslant|t| \leqslant 1 \\ 0 & \text { otherwise }\end{cases}
$$

after orthonormalization. We have the following result, when the convergence assumption of Theorem 3.5 is modified to hold for each $f_{m}$ in (4.2). 
Theorem 4.2. Let $F$ be an even function for which $F$ and $\check{F}$ are in $L^{1}(\mathbb{R}) \cap$ $L^{2}(\mathbb{R})$. Then, $F$ can be written as

$$
F(\gamma)=\sum_{m=0}^{\infty} \sum_{n=1}^{\infty} K_{m, n} \frac{n}{2^{m}}\left(\frac{\sin \pi \frac{n}{2^{m}} \gamma}{\pi \frac{n}{2^{m}} \gamma}\right)^{2},
$$

where the convergence is in $L^{2}(\widehat{\mathbb{R}})$ norm.

Proof. (i) Given $F \in L^{2}(\widehat{\mathbb{R}})$, one can write its inverse Fourier transform as

$$
f=\check{F}=\sum_{m=0}^{\infty} f_{m},
$$

where each $f_{m}$ is in $V_{m}$. This follows from the discussion on the Battle-Lemarié wavelets in [12] for the case where the scaling function is obtained from

$$
\phi(t)= \begin{cases}1-|t| & 0 \leqslant|t| \leqslant 1 \\ 0 & \text { otherwise }\end{cases}
$$

(ii) Each $f_{m}$ is piecewise linear on the intervals $\left[2^{-m} k, 2^{-m}(k+1)\right]_{k \in \mathbb{Z}}$. If, in addition, $F$ is even (which makes $\check{F}$ even), then each $f_{m}$ is also even. As mentioned in Definition 4.1 we can write each $f_{m}$ as a linear combination of the triangles $\triangle_{k}^{(m)}(t)=\max \left(1-\frac{2^{m}|t|}{k}, 0\right)$. Thus, we have

$$
f_{m}(t)=\sum_{n=1}^{\infty} K_{m, n} \max \left(1-\frac{2^{m}|t|}{n}, 0\right),
$$

and so

$$
\check{F}(t)=\sum_{m=0}^{\infty} \sum_{n=1}^{\infty} K_{m, n} \max \left(1-\frac{2^{m}|t|}{n}, 0\right) .
$$

This is a double indexed family of dilations of the triangles, where $n$ corresponds to translation of the scaling function $\phi$ and $m$ corresponds to dilation. Note that $\phi=h_{0}$, and, as mentioned in Remark 3.7, the translations of $\phi$ are transformed into dilations of the triangles $\max \left(1-\frac{2^{m}|t|}{n}, 0\right)$ giving rise to the second dilation. The convergence in (4.3) is in the $L^{2}$ sense.

(iii) Also, if $\check{F} \in L^{1}(\mathbb{R}) \cap L^{2}(\mathbb{R})$, then one can justify interchanging the infinite sums in the following integral to obtain

$$
\begin{aligned}
F(\gamma) & =\int_{\mathbb{R}}\left(\sum_{m=0}^{\infty} \sum_{n=1}^{\infty} K_{m, n} \max \left(1-\frac{2^{m}|t|}{n}, 0\right)\right) e^{-2 \pi i t \gamma} d t \\
& =\sum_{m=0}^{\infty} \sum_{n=1}^{\infty} \int_{\mathbb{R}} K_{m, n} \max \left(1-\frac{2^{m}|t|}{n}, 0\right) e^{-2 \pi i t \gamma} d t \\
& =\sum_{m=0}^{\infty} \sum_{n=1}^{\infty} K_{m, n} \frac{n}{2^{m}}\left(\frac{\sin \pi \frac{n}{2^{m}} \gamma}{\pi \frac{n}{2^{m}} \gamma}\right)^{2} .
\end{aligned}
$$


Example 4.3. Theorem 4.2 can be formulated from the point of view of sampling in the following way, cf. Remark 3.8. Ultimately we would like to replace the constants $K_{m, n}$ by some expression in terms of the sampled values of $F$. Motivated by our discussion on multiresolution analysis, we first write the $f_{m} \mathrm{~s}$ in (4.2) in terms of hat functions $h_{k}^{(m)}$. In fact, each $f_{m}$ can be written as

$$
f_{m}=\sum_{n=-\infty}^{\infty} f_{m}\left(\frac{n}{2^{m}}\right) h_{n}^{(m)} .
$$

If $F$ is even then let $H_{1}^{(m)}=h_{0}^{(m)}$ and for $n>0$ let $H_{n+1}^{(m)}=h_{n}^{(m)}+h_{-n}^{(m)}$. We have

$$
f_{m}=\sum_{n=1}^{\infty} f_{m}\left(\frac{n-1}{2^{m}}\right) H_{n}^{(m)}
$$

which means

$$
\check{F}=\sum_{m=0}^{\infty} \sum_{n=1}^{\infty} f_{m}\left(\frac{n-1}{2^{m}}\right) H_{n}^{(m)} .
$$

As shown in (3.4) the function $H_{n}^{(m)}$ can be written in terms of the first $n$ triangles, $\triangle_{k}^{(m)}(t)$, see Example 4.1. This gives

$$
\check{F}(t)=\sum_{m=0}^{\infty} \sum_{n=1}^{\infty} \sum_{k=1}^{n} c_{k} f_{m}\left(\frac{n-1}{2^{m}}\right) \max \left(1-\frac{2^{m}|t|}{k}, 0\right),
$$

and so

$$
F(\gamma)=\sum_{m=0}^{\infty} \sum_{n=1}^{\infty} \sum_{k=1}^{n} c_{k} f_{m}\left(\frac{n-1}{2^{m}}\right) \frac{k}{2^{m}}\left(\frac{\sin \pi \frac{k}{2^{m}} \gamma}{\pi \frac{k}{2^{m}} \gamma}\right)^{2} .
$$

Note that the constants $c_{k}$ can be obtained from (3.4) and what appears in the proof of Proposition 3.4. Thus, the function $F$ can be represented in terms of the samples of the projections of $\check{F}$ on the spaces $V_{m}$.

Example 4.4. Consider the function $F \in A(\widehat{\mathbb{R}}) \cap L^{1}(\widehat{\mathbb{R}}) \cap L^{2}(\widehat{\mathbb{R}})$ with inverse Fourier transform given by the continuous trapezoid function,

$$
\check{F}=\left\{\begin{array}{cc}
a & \text { on }[-1,1], \text { where } a \text { is some positive constant } \\
\text { linear } & \text { on }[-2,-1] \text { and }[1,2] \\
0 & \text { otherwise }
\end{array}\right.
$$

where $\operatorname{supp} \check{F}=[-2,2] . \check{F}$ is even and belongs to the space $A(\mathbb{R}) \cap L^{1}(\mathbb{R}) \cap L^{2}(\mathbb{R})$. Here we can just work on the space $V_{0}$, and so, by (4.4) for just $m=0$, we have

$$
\check{F}=f_{0}=a h_{0}+a\left(h_{-1}+h_{1}\right)=a H_{1}+a H_{2} .
$$


From (3.4) this can be further written as

$$
\check{F}=a \triangle_{1}+a 2\left(\triangle_{2}-\triangle_{1}\right)=-1 f_{0}(0) \triangle_{1}+2 f_{0}(1) \triangle_{2} .
$$

After taking the Fourier transform we obtain

$$
\begin{aligned}
F(\gamma) & =(-1) f_{0}(0)\left(\frac{\sin \pi \gamma}{\pi \gamma}\right)^{2}+2 f_{0}(1) 2\left(\frac{\sin \pi 2 \gamma}{\pi 2 \gamma}\right)^{2} \\
& =(-1) \check{F}(0)\left(\frac{\sin \pi \gamma}{\pi \gamma}\right)^{2}+4 \check{F}(1)\left(\frac{\sin \pi 2 \gamma}{\pi 2 \gamma}\right)^{2}
\end{aligned}
$$

cf., [30].

\section{Conclusion}

Bounded sequences have been constructed on $\mathbb{Z}$ whose autocorrelations at integers are inverse Fourier transforms of linear combinations of dilates of Fejér functions. In the setting of $\mathbb{R}$ this motivated a decomposition formula for expressing any even function in $L^{1}(\mathbb{R}) \cap L^{2}(\mathbb{R})$ as a doubly indexed sum of dilates of the Fejér function. One of the indices represents scaling while the other represents multiplication, and this gives the multiplicative MRA structure to the decomposition.

\section{ACKNOWLEDGEMENT}

The first named author gratefully acknowledges support from ONR Grant N0001409-1-0144, AFOSR Grant FA9550-05-1-0443, and ARO Grant W911NF-09-10383. The second named author gratefully acknowledges support from NSFDMS Grant 0914892 and DARPA Grant HR0011-09-1-0033. Finally, we want to thank the two referees for their thorough and constructive reviews.

\section{References}

[1] L. Auslander and P. E. Barbano, Communication codes and Bernoulli transformations, Appl. Comput. Harmon. Anal., 5(2), 109-128, 1998.

[2] J. J. Benedetto, A multidimensional Wiener-Wintner theorem and spectrum estimation, Trans. Amer. Math. Soc., 327(2), 833-852, 1991.

[3] _ Harmonic Analysis and Applications, CRC Press, Boca Raton, FL, 1997.

[4] J. J. Benedetto and S. Datta, Construction of infinite unimodular sequences with zero autocorrelation, -it Advances in Computational Mathematics, 32(2), 191 - 207, 2010. 
[5] J. J. Benedetto and J. J. Donatelli, Ambiguity function and frame theoretic properties of periodic zero autocorrelation waveforms, IEEE J. Special Topics Signal Processing, 1, 6-20, 2007.

[6] J. J. Benedetto, I. Konstantinidis, and M. Rangaswamy, Phase-coded waveforms and their designs, IEEE Signal Processing Magazine, 26, 22-31, 2009.

[7] John J. Benedetto and Paulo J. S. G. Ferreira (eds.), Modern Sampling Theory, Applied and Numerical Harmonic Analysis, Birkhäuser Boston, Boston, MA, 2001.

[8] P. L. Butzer and R. L. Stens, Sampling theory for not necessarily bandlimited functions: A historical overview, SIAM Review, 34(1), 40-53, 1992.

[9] O. Christensen, An Introduction to Frames and Riesz Bases, Birkhäuser, 2003.

[10] C.K. Chui (ed.), Wavelets: A Tutorial in Theory and Applications, Wavelet Analysis and its Applications, vol. 2, Academic Press, 1992.

[11] S. Datta, Wiener's Generalized Harmonic Analysis and Waveforem Design, Ph.D. thesis, University of Maryland, College Park, Maryland, 2007.

[12] I. Daubechies, Ten Lectures on Wavelets, SIAM, 1992.

[13] C. de Boor, K. Höllig and S. Riemenschneider, Box Splines, Springer-Verlag, 1993.

[14] P. J. S. G. Ferreira, Approximating non-band-limited functions by nonuniform sampling series, 1995 Workshop on Sampling Theory and Applications, 276-281, 1995.

[15] P.J.S.G. Ferreira, Nonuniform sampling of nonbandlimited signals, IEEE Signal Processing Letters, 2(5), 89-91, 1995.

[16] T. Helleseth and P. V. Kumar, Sequences with low correlation, Handbook of coding theory, Vol. I, II, North-Holland, Amsterdam, 1765-1853, 1998.

[17] R. Kerby, The Correlation Function and the Wiener-Wintner Theorem in Higher Dimension, Ph.D. thesis, University of Maryland, College Park, 1990.

[18] , personal communication, 2008.

[19] N. Levanon and E. Mozeson, Radar Signals, Wiley Interscience, IEEE Press, 2004.

[20] M. L. Long, Radar Reflectivity of Land and Sea, Artech House, 2001. 
[21] F. Marvasti, Nonuniform Sampling: Theory and Practice, Springer Verlag, New York, 2001.

[22] W. H. Mow, A new unified construction of perfect root-of-unity sequences, Proc. IEEE 4th International Symposium on Spread Spectrum Techniques and Applications (Germany), 955-959, September 1996.

[23] F. E. Nathanson, Radar Design Principles - Signal Processing and the Environment, SciTech Publishing Inc., Mendham, NJ, 1999.

[24] W. Rudin, Fourier Analysis on Groups, Interscience Tracts in Pure and Applied Mathematics, No. 12, Interscience Publishers (a division of John Wiley and Sons), New York-London, 1962.

[25] E. M. Stein and G. Weiss, Introduction to Fourier Analysis on Euclidean Spaces, Princeton University Press, Princeton, N.J., 1971.

[26] G. W. Stimson, Introduction to Airborne Radar, SciTech Publishing Inc., Mendham, NJ, 1998.

[27] S. Ulukus and R. D. Yates, Iterative construction of optimum signature sequence sets in synchronous CDMA systems, IEEE Trans. Inform. Theory, 47(5),1989-1998, 2001.

[28] S. Verdú, Multiuser Detection, Cambridge University Press, Cambridge, UK, 1998.

[29] N. Wiener, Generalized harmonic analysis, Acta Math., no. 55, 117-258, 1930.

[30] _ The Fourier Integral and Certain of its Applications, Cambridge Mathematical Library, Cambridge University Press, Cambridge, 1988, Reprint of the 1933 edition. With a foreword by Jean-Pierre Kahane.

[31] N. Wiener and A. Wintner, On singular distributions, J. Math. Phys., 233246, 1939. 